\title{
Special Column on Multiscale Stochastic Finite Element Method
}

In the past decade, multi-scale modelling in mathematical physics and engineering has been growing with vast advancement based on deterministic assumption. Because all information across scales contains a certain level of uncertainty due to incomplete knowledge and inherent chaos with respect to physical laws and parameters, a combination of multi-scale and stochastic techniques becomes a new approach. Incorporation of stochastic process into multi-scale modelling with accurate assessment of uncertainty propagation across scales is highly challenging but essential for a rising number of applications, and in certain areas such models can provide critical insights that deterministic approaches could never predict. In computational mechanics, research topics on discrete-to-continuum, stochastic-to-deterministic, and scale coupling are prioritized as the most significant directions. In the past half century, the finite element method has been a mature and widely-used numerical technique. When both multi-scale and stochastic phenomena are considered, cross-cutting multi-scale stochastic finite element modelling forms a novel research frontier. In this special issue, there are five original research papers relevant to multi-scale stochastic finite element methods or their applications.

The first paper by $X i$ Frank $X u$ puts its focus on the investigation of a fast multiscale stochastic finite element computing procedure on random field modeling of geotechnical problems. A unique fast computing advantage of the procedure enables computation performed only on those locations of interest, therefore saving a lot of computation. The numerical example on soil settlement shows that the procedure achieves significant computing efficiency compared with Monte Carlo method. The example shows that using mean value of the soil elastic properties underestimates thesettlement, about $14 \%$ in this case. It is remarked that MSFEM can achieve further computing saving by using numerical truncation scheme on Green function and the autocorrelation function. Further results will be reported on the computing saving and computation of variance.

George Stefanou demonstrates that a particular kind of non-Gaussian random fields with great practical importance is that of translation fields resulting from a simple memory-less transformation of an underlying
Gaussian field with known second-order statistics. This paper provides a critical examination of existing random field models of heterogeneous two-phase media with emphasis on level-cut random fields which are a special case of translation fields. The case of random level sets, often used to represent the geometry of physical systems, is also examined. Two numerical examples are provided to illustrate the basic features of the different approaches.

Sakata et al. discusse a multiscale stochastic analysis of a laminated composite plate consisting of unidirectional fiber reinforced composite laminae. In particular, influence of a microscopic random variation of the elastic properties of component materials on mechanical properties of the laminated plate is investigated. This study deals with the stochastic response of a laminated composite plate against the microscopic random variation in addition to a random variation of fiber orientation in each lamina, and stochastic properties of the mechanical responses of the laminated plate is investigated. Halpin-Tsai formula and the homogenization theory-based finite element analysis are employed for estimation of effective elastic properties of lamina, and the classical laminate theory is employed for analysis of a laminated plate.

Bruno Sudret et al. present the spatial variability of stress fields resulting from polycrystalline aggregate calculations involving random grain geometry and crystal orientations is investigated. A periodogrambased method is proposed to identify the properties of homogeneous Gaussian random fields (power spectral density and related covariance structure). Based on a set of finite element polycrystalline aggregate calculations the properties of the maximal principal stress field are identified. Two cases are considered, using either a fixed or random grain geometry. The stability of the method w.r.t the number of samples and the load level (up to $3.5 \%$ macroscopic deformation) is investigated.

The final paper by Si Salem et al. investigates a new concrete-composite beam with high mechanical performances to weight ratio. The proposed design technique consists to embed a cylindrical polymer tube wrapped by a GFRP Jacket in the mechanically ineffective concrete tensile zone. An experimental investigation is carried out on composite beams under bending loads until failure to evaluate the flexural capacity and the corresponding 
failure mechanisms. Based on the experimental results, statistical and preliminary reliability analyses using the FORM method are performed to assess the safety margin of the new beam.

Acknowledgements This special column was financially supported by the Project for Enhancing International Impact of China STM Journals 2013.

\section{Guest Editors,}

Xi F. XU

Professor, Beijing Jiaotong University, China

George STEFANOU

Assistant Professor, Aristotle University of Thessaloniki, Greece

Carsten PROPPE

Professor, Karlsruhe Institute of Technology, Germany

\section{Executive Editor,}

Yu Ching WU

Associate Professor, Tongji University, China

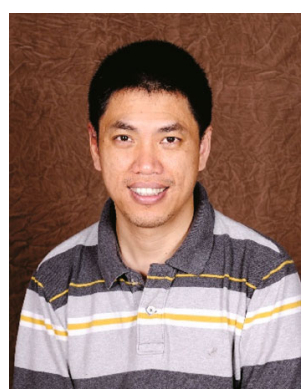

Xi F. XU received a Bachelor degree from Tsinghua University, China in 1993. He had practiced as a structural engineer for 5 years in Shanghai before returning to graduate school. He received two Master degrees, one in offshore engineering from Robert Gordon University, and the other in civil engineering from University of Akron, USA. In 2005 he completed his PhD degree in civil engineering from Johns Hopkins and then served as an assistant professor at Stevens Institute of Technology, USA. $\mathrm{He}$ is now a professor at Beijing Jiaotong University, China. His research interests cover a variety of topics in the areas of engineering reliability, multiscale mechanics, micromechanics, and stochastic dynamics. He is one of pioneering researchers in promoting development of multiscale methods onto stochastic systems.

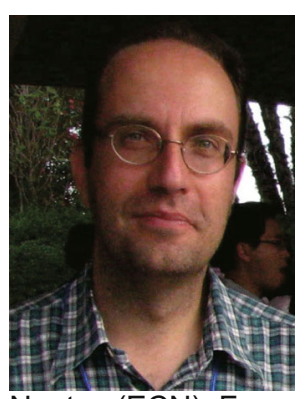

George STEFANOU graduated from the School of Civil Engineering of the National Technical University of Athens (NTUA), Greece. He received his postgraduate and $\mathrm{PhD}$ degrees in the field of Computational Stochastic Mechanics from NTUA. After having worked in 2008 as a post-doctoral researcher at the Ecole Centrale de Nantes (ECN), France, he had a two-year research collaboration with the Department of Civil Engineering and Engineering Mechanics at Columbia University in the City of New York, USA (2012-2013). He is currently an Assistant Professor at the Department of Civil Engineering of the Aristotle University of Thessaloniki. His teaching includes basic and advanced courses in structural analysis with the finite element method. His research activity is mainly focused on the development and application of computer methods for stochastic finite element analysis of real world structures as well as on the multiscale modeling and uncertainty quantification of heterogeneous materials and structures. He has been involved in the following scientific activities: Guest Editor of 2 special issues of international scientific journals, member of the Scientific Committee of 7 international conferences, reviewer for 38 scientific journals and 17 international conferences.

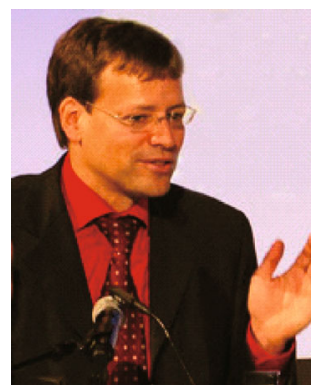

Carsten PROPPE received in 1995 Dipl.-Ing., Dipl.-Math from Engineering and Mathematics at TU Berlin and Ecole Centrale de Lyon, Germany. In 1999, he has got a doctoral degree from Institut für Mechanik, Otto-vonguericke-universität, magdeburg, Germany, focused on Engineering Mechanics of Random Vibrations. From 1999 to 2002, he has been an assistant professor in Institut für Mechanik, Leopold-Franzens-Universität, Innsbruck. After having worked as an Engineer on Vehicle Dynamics in Bombardier Transportation $\mathrm{GmbH}$ for several years, he has been a professor of Engineering Mechanics at Universität Karlsruhe in Germany since 2005. His research interests cover a variety of topics in the areas of multibody dynamics, structural dynamics, multiscale computing, uncertainty quantification, etc. 